\title{
BOUNDED APPROXIMATE IDENTITIES IN THE ALGEBRA OF COMPACT OPERATORS ON A BANACH SPACE
}

\author{
CHRISTIAN SAMUEL \\ (Communicated by Palle E. T. Jorgensen)
}

\begin{abstract}
We characterize Banach spaces $X$ for which the algebra of compact operators $\mathscr{K}(X)$ admits approximate bounded right identities.
\end{abstract}

\section{INTRODUCTION}

In his monograph [ $\mathrm{J}]$ Johnson asked if, for every Banach space $X$, the algebra $\mathscr{K}(X)$ of all compact operators on $X$ is amenable. It is known that an amenable Banach algebra admits bounded approximate identities and that there exists a Banach space $X$ such that the algebra $\mathscr{K}(X)$ does not admit any bounded left approximate identity [D]. Therefore, the answer to Johnson's question is no. It is interesting to study connections between amenability of the algebra $\mathscr{K}(X)$ and property of structure of $X$. In this paper we characterize Banach spaces $X$ for which $\mathscr{K}(X)$ admits bounded right approximate identities. We show that if $\mathscr{K}(X)$ admits a bounded right approximate identity then it admits a bounded left approximate identity. An example of a Banach space $X$ for which $\mathscr{K}(X)$ admits a bounded left approximate identity and no bounded right approximate identity is given.

\section{NOTATION AND PRELIMINARIES}

If $X$ is a Banach space, $\mathscr{K}(X)$ will denote the algebra of all compact operators on $X$ and $B_{X}$ will denote the closed unit ball of $X$. All the operators are assumed to be continuous.

Let $\lambda \geq 1$. A Banach space $X$ is said to have the $\lambda$-bounded compact approximation property ( $\lambda$-b.c.a.p. in short) if for every finite subset $F \subset X$ and for every $\varepsilon>0$ there is an operator $u \in \mathscr{K}(X)$ such that $\|u\| \leq \lambda$ and $\|u(x)-x\| \leq \varepsilon$ for all $x \in F$. The space is said to have the bounded compact approximation property (b.c.a.p. in short) if it has the $\lambda$-b.c.a.p. for a $\lambda \geq 1$.

Let $\lambda \geq 1$. The dual $X^{*}$ of a Banach space $X$ is said to have the $* \lambda$-bounded compact approximation property ( $* \lambda$-b.c.a.p. in short) if for every finite subset $F \subset X^{*}$ and for every $\varepsilon>0$ there is an operator $u \in \mathscr{K}(X)$ such that $\|u\| \leq \lambda$

Received by the editors March 29, 1991 and, in revised form, August 1, 1991.

1991 Mathematics Subject Classification. Primary 46B20, 47B05.

Key words and phrases. Bounded compact approximation property, approximate identity, compact operators. 
and $\left\|u^{*}\left(x^{*}\right)-x^{*}\right\| \leq \varepsilon$ for all $x^{*} \in F$. The space is said to have the $*$-bounded compact approximation property (*-b.c.a.p. in short) if it has the $* \lambda$-b.c.a.p. for a $\lambda \geq 1$.

A net $\left(u_{\alpha}\right)_{\alpha}$ of a Banach algebra $A$ is a bounded right (left) approximate identity if $\sup _{\alpha}\left\|u_{\alpha}\right\|<+\infty$ and $\lim _{\alpha} a u_{\alpha}=a\left(\lim _{\alpha} u_{\alpha} a=a\right)$ for every $a \in$ $A$. A net which is a bounded left approximate identity and a bounded right approximate identity is a bounded approximate identity.

\section{BOUNDED APPROXIMATE IDENTITIES}

Theorem 1. If $X$ is a Banach space such that $X^{*}$ has the *-b.c.a.p. then $X$ has the b.c.a.p.

Proof. Let $\lambda \geq 1$ such that $X^{*}$ has the $* \lambda$-b.c.a.p.; there exists a net $\left(u_{\alpha}\right)_{\alpha \in \mathscr{A}}$ of $\mathscr{K}(X)$ such that $\sup _{\alpha}\left\|u_{\alpha}\right\| \leq \lambda$ and $\lim _{\alpha}\left\|u_{\alpha}^{*}\left(x^{*}\right)-x^{*}\right\|=0$ for every $x^{*} \in X^{*}$. Then we have $u_{\alpha}(x) \rightarrow x$ weakly for every $x \in X$. Let $F$ be a finite subset of $X$ and $\varepsilon>0$. There exists a convex combination $v$ of the $\left\{u_{\alpha} ; \alpha \in \mathscr{A}\right\}$ such that $\|v(x)-x\| \leq \varepsilon$ for every $x \in F$; of course, $\|v\| \leq \lambda$ and $v \in \mathscr{K}(X)$.

Remark 1. Let $X$ be a reflexive Banach space. Then the following properties are equivalent:

(a) $X$ has the b.c.a.p.,

(b) $X^{*}$ has the b.c.a.p.,

(c) $X^{*}$ has has the $*$-b.c.a.p.

Remark 2. There exists a Banach space $Z$ with the b.c.a.p. such that $Z^{*}$ does not have the *-b.c.a.p. We know that there exists a separable reflexive Banach space $Y$ without the b.c.a.p. [LT]. Using the result of Lindenstrauss [L] there exists a Banach space $Z$ with a boundedly complete basis such that $Z^{*}$ has a complemented subspace isomorphic to $Y^{*}$. Every complemented subspace of a Banach space with the b.c.a.p. has the b.c.a.p., and so $Z^{*}$ does not have the b.c.a.p.; it is then obvious that $Z^{*}$ does not have the $*$-b.c.a.p.

Theorem 2. Let $X$ be a Banach space. Then $\mathscr{K}(X)$ admits a bounded right approximate identity if and only if $X^{*}$ has the *-b.c.a.p.

Proof. (i) Let $X$ be a Banach space such that $\mathscr{K}(X)$ admits a bounded right approximate identity $\left(v_{\beta}\right)_{\beta \in \mathscr{B}}$ and $\sup _{\beta}\left\|v_{\beta}\right\| \leq \lambda$. Let $F$ be a finite subset of $X^{*}$. Then there exists a projection on the finite-dimensional subspace spanned by $F$. According to Lemma 3.1 of [JRZ] there exists a finite rank projection $\pi$ on $X$ such that $\pi^{*}\left(x^{*}\right)=x^{*}$ for every $x^{*} \in F$. We have $\lim _{\beta}\left\|\pi \circ v_{\beta}-\pi\right\|=0$ so $\lim _{\beta}\left\|v_{\beta}^{*} \circ \pi^{*}-\pi^{*}\right\|=0$ and then for every $x^{*} \in F$ we have

$$
x^{*}=\pi^{*}\left(x^{*}\right)=\lim _{\beta} v_{\beta}^{*}\left(\pi^{*}\left(x^{*}\right)\right)=\lim _{\beta} v_{\beta}^{*}\left(x^{*}\right) .
$$

(ii) Let $X$ be a Banach space such that $X^{*}$ has the $* \lambda$-b.c.a.p. There exists a net $\left(u_{\alpha}\right)_{\alpha \in \mathscr{A}}$ on $\mathscr{K}(X)$ such that $\sup _{\alpha}\left\|u_{\alpha}\right\| \leq \lambda$ and $\lim _{\alpha}\left\|u_{\alpha}^{*}\left(x^{*}\right)-x^{*}\right\|=0$ for every $x^{*} \in X^{*}$. We shall show that $\left(u_{\alpha}\right)_{\alpha \in \mathscr{A}}$ is a bounded right approximate identity on $\mathscr{K}(X)$. Let $u \in \mathscr{K}(X), \varepsilon>0$, and $\varepsilon^{\prime}>0$ such that $(\lambda+3) \varepsilon^{\prime} \leq \varepsilon$. $u^{*}$ is a compact operator on $X^{*}$; then $u^{*}\left(B_{X^{*}}\right)$ is a totally bounded subset of $X^{*}$. There exists a finite subset $F \subset u^{*}\left(B_{X^{*}}\right)$ such that

$$
\min \left\{\left\|u^{*}\left(x^{*}\right)-y^{*}\right\| ; y^{*} \in F\right\} \leq \varepsilon^{\prime}
$$


for every $x^{*} \in B_{X^{*}}$. There exists $\alpha_{0} \in \mathscr{A}$ such that $\left\|u_{\alpha}^{*}\left(y^{*}\right)-y^{*}\right\| \leq \varepsilon^{\prime}$ for every $\alpha \succeq \alpha_{0}$ and for every $y^{*} \in F$. Let $x^{*} \in B_{X^{*}}$ and $y^{*} \in F$ such that $\left\|u^{*}\left(x^{*}\right)-y^{*}\right\| \leq \varepsilon^{\prime}$. For $\alpha \succeq \alpha_{0}$ we have

$$
\begin{aligned}
\left\|u^{*}\left(x^{*}\right)-u_{\alpha}^{*} \circ u^{*}\left(x^{*}\right)\right\| & \leq\left\|u^{*}\left(x^{*}\right)-y^{*}\right\|+\left\|y^{*}-u_{\alpha}^{*}\left(y^{*}\right)\right\|+\left\|u_{\alpha}\left(y^{*}-u^{*}\left(x^{*}\right)\right)\right\| \\
& \leq \varepsilon^{\prime}+\varepsilon^{\prime}+\lambda \varepsilon^{\prime}=(\lambda+3) \varepsilon^{\prime} \leq \varepsilon,
\end{aligned}
$$

and so $\left\|u^{*}-u_{\alpha}^{*} \circ u^{*}\right\|=\left\|u-u \circ u_{\alpha}\right\| \leq \varepsilon$.

Remark 3. Dixon has shown in [D] that a Banach space $X$ has the b.c.a.p. if and only if $\mathscr{K}(X)$ admits a bounded left approximate identity. Using Theorems 1 and 2 we deduce that $\mathscr{K}(X)$ admits a bounded left approximate identity if $\mathscr{K}(X)$ admits a bounded right approximate identity.

Remark 4. The algebra $\mathscr{K}(Z)$ of compact operators on the space $Z$ introduced in Remark 2 admits a bounded left approximate identity and no bounded right approximate identity.

Remark 5. Let $X$ be a reflexive Banach space; $\mathscr{K}(X)$ admits a bounded left approximate identity if and only if $\mathscr{K}(X)$ admits a bounded right approximate identity; $\mathscr{K}(X)$ admits a bounded approximate identity if and only if $\mathscr{K}\left(X^{*}\right)$ admits a bounded approximate identity.

Remark 6. Let $X$ be a Banach space such that $\mathscr{K}(X)$ is an $M$-ideal in the algebra of all operators on $X$. Then $\mathscr{K}(X)$ admits a bounded approximate identity $\left(T_{\alpha}\right)_{\alpha}$ such that $\sup _{\alpha}\left\|T_{\alpha}\right\| \leq 1$ and $\lim _{\alpha}\left\|I-T_{\alpha}\right\|=1$ [HL].

\section{OPEN PROBLEMS}

The author expresses his gratitude to the referee for his valuable open problems.

Let us recall that a Banach space $X$ is said to have the bounded approximation property (b.a.p. in short) if there exists $\lambda \geq 1$ such that for every $\varepsilon>0$ and for every finite subset $F \subset X$ there exists a finite rank operator $u$ in $X$ such that $\|u\| \leq \lambda$ and $\|u(x)-x\| \leq \varepsilon$ for all $x \in F$.

Question 1. If $X$ has the b.c.a.p., does $X$ have the b.a.p.? At least what additional property of $X$ is needed to ensure that the b.c.a.p. implies the b.a.p.?

Question 2. If $X^{*}$ has the b.c.a.p., does $X^{*}$ have the *-b.c.a.p., or at least, does $X$ have the b.c.a.p.? Of course if the first part of Question 1 has an affirmative answer then so does the second part of Question 2.

A Banach space $X$ is an Asplund space if every separable subspace of $X$ has a separable dual. It is known [G] that if $X$ is reflexive (or more generally, the dual of an Asplund space) and has the b.a.p. then it has the 1-b.a.p.

Question 3. If $X$ is reflexive (or more generally, the dual of an Asplund space) and has the b.c.a.p, does $X$ have the 1-b.c.a.p.?

\section{REFERENCES}

[D] P. G. Dixon, Left approximate identities in algebras of compact operators on Banach spaces, Proc. Roy. Soc. Edinburgh Sect. A 104 (1986), 169-175.

[G] A. Grothendieck, Produits tensoriels topologiques et espaces nucléaires, Mem. Amer. Math. Soc., no. 16, Amer. Math. Soc., Providence, RI, 1955. 
[J] B. E. Johnson, Cohomology in Banach algebras, Mem. Amer. Math. Soc., no. 127, Amer. Math. Soc., Providence, RI, 1972.

[JRZ] W. B. Johnson, H. P. Rosenthal, and M. Zippin, On bases, finite dimensional decompositions and weaker structures in Banach spaces, Israel J. Math. 9 (1971), 488-506.

[HL] P. Harmand and A. Lima, Banach spaces which are M-ideals in their biduals, Trans. Amer. Math. Soc. 283 (1984), 253-264.

[L] J. Lindenstrauss, On James's paper "Separable conjugate spaces", Israel J. Math. 9 (1971), 279-284.

[LT] J. Lindenstrauss and L. Tzafriri, Classical Banach spaces. I, Springer-Verlag, Berlin and New York, 1977.

Institut de Calcul Mathématique iCM, CNRS (URA 225) \& Mathématiques, Faculté des Sciences et TechniQues de Saint-Jérôme, 13397 Marseille Cedex 13, France

E-mail address: samuel@frmrs11.bitnet 